\title{
Boostream: a dynamic fluid flow process to assemble nanoparticles at liquid interface
}

Olivier Delléa* and Olivier Lebaigue

Grenoble Alpes University, CEA-LITEN, 17 rue des Martyrs, 38054 Grenoble Cedex 09, France

Received: 17 April 2017 / Accepted: 31 May 2017

\begin{abstract}
CEA-LITEN develops an original process called Boostream ${ }^{\circledR}$ to manipulate, assemble and connect micro- or nanoparticles of various materials, sizes, shapes and functions to obtain monolayer colloidal crystals (MCCs). This process uses the upper surface of a liquid film flowing down a ramp to assemble particles in a manner that is close to the horizontal situation of a Langmuir-Blodgett film construction. In presence of particles at the liquid interface, the film down-flow configuration exhibits an unusual hydraulic jump which results from the fluid flow accommodation to the particle monolayer. In order to master our process, the fluid flow has been modeled and experimentally characterized by optical means, such as with the moiré technique that consists in observing the reflection of a succession of periodic black-and-red fringes on the liquid surface mirror. The fringe images are deformed when reflected by the curved liquid surface associated with the hydraulic jump, the fringe deformation being proportional to the local slope of the surface. This original experimental setup allowed us to get the surface profile in the jump region and to measure it along with the main process parameters (liquid flow rate, slope angle, temperature sensitive fluid properties such as dynamic viscosity or surface tension, particle sizes). This work presents the experimental setup and its simple model, the different experimental characterization techniques used and will focus on the way the hydraulic jump relies on the process parameters.
\end{abstract}

Keywords: Nanoparticles / Langmuir-Blodgett / liquid interface / hydraulic jump / moiré

\section{Introduction}

Monolayer colloidal crystals (MCCs) are a monolayer of close-packed monodisperse colloidal micro- or nanospheres having hexagonal symmetry in the plane [1] (Fig. 1). Materials and surfaces templated by MCCs impact numerous domain such as photovoltaics for light trapping [2], optical sensors to detect physical variations through structural color changes [3-5] (Fig. 2), mechanics to change surface friction coefficient and wettability [6].

Today, many different techniques such as LangmuirBlodgett [7], spin coating [8], capillary force assembly [9,10] and electrostatics-assisted layer-by-layer assembly [11] exist to fabricate MCCs via self-assembly of monodisperse microspheres. However, the limitations of these techniques are mainly the lack of control during the assembly process, the lack of scalability needed for mass production, noncompatibility with 3D or non-planar surfaces.

In this context, an original process called Boostream ${ }^{\circledR}$ [12] inspired by previous works [13] has been developed at CEA to assemble micro- or nanoparticles of various materials, sizes, shapes and functions allowing the

\footnotetext{
* e-mail: olivier.dellea@cea.fr
}

fabrication of ordered structures as MCCs. This process is similar to the Langmuir-Blodgett technique but uses a liquid film flowing down a ramp to assemble particles at the liquid interface to form a compact film. The film floating on the liquid flowing down gives rise to specific hydraulic behaviors as an unusual hydraulic jump resulting from the fluid flow accommodation.

In this paper, we describe the experimental setup and optical experimental techniques used to characterize flow dynamic. We present a theoretical approach to study the relationship between hydraulic jump and process parameters. We investigate the role played by capillary forces and viscous shear stresses at the liquid interface on particle assembly.

\section{Experimental section}

\subsection{Experimental setup}

The basic principle of Boostream ${ }^{\circledR}$ process is shown in Figure 3. A colloidal suspension is spread on a moving liquid flowing down a ramp. The angle $\alpha$ of the ramp is kept constant at $11^{\circ}$. The liquid is deionized (DI) water at room temperature with a flow rate varying from 100 to $900 \mathrm{~mL} /$ min. Colloids dispensed at the surface of the liquid are 

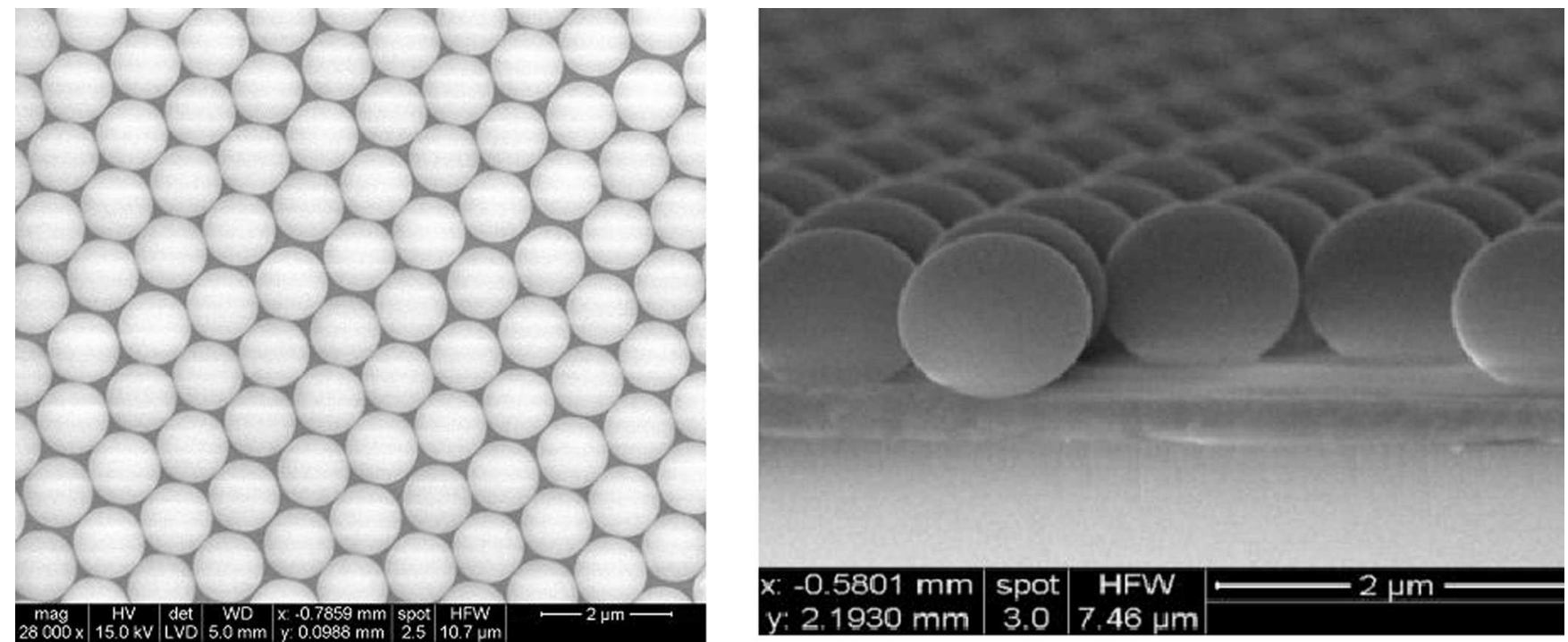

Fig. 1. SEM images of MCCs constituted of silica sphere $(\varnothing=1 \mu \mathrm{m})$ deposited on silicon substrate, in top view (left) and in cross section view (right).

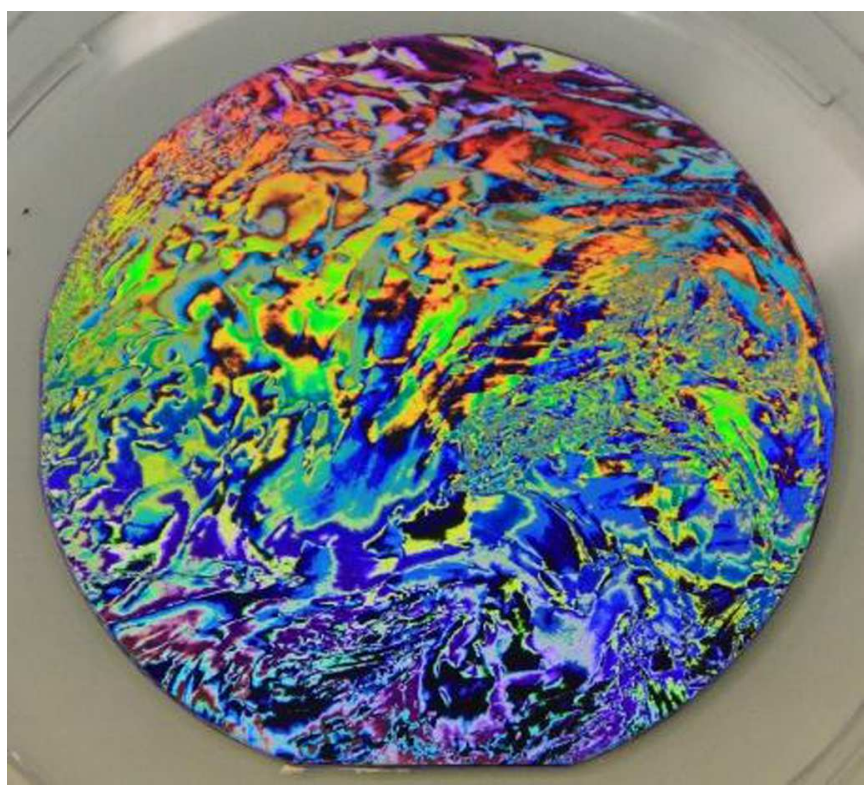

Fig. 2. View of structural colors generated by a monolayer colloidal crystal of silica spheres $(\varnothing=1 \mu \mathrm{m})$ deposited on a $4 \prime \prime$ silicon substrate and illuminated with non-collimated white light source.

trapped at the air-liquid interface and driven by hydrodynamic forces to the area called "transfer zone". The particles gradually accumulated at the liquid interface lead to their self-assembly and the formation in the transfer zone and at the beginning of the ramp of a compact monolayer film.

Even if the particles are microscopic, the film forming due to the packing up of particles is generally visible to the naked eye because of diffraction, diffusion or interference phenomena (Fig. 4). The compact film floating at the liquid surface could be transferred on a substrate previously immersed in the transfer zone by applying a slow withdrawing movement (few $\mathrm{cm} / \mathrm{min}$ ).

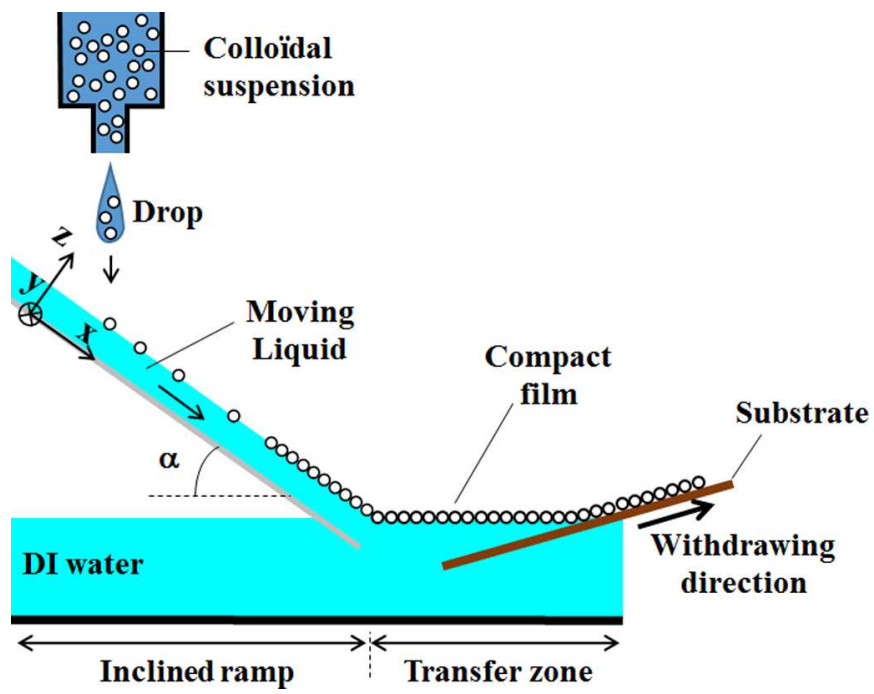

Fig. 3. Boostream ${ }^{\circledR}$ process.

\subsection{Materials and suspension preparation}

The colloid suspension is composed of monodisperse silica microspheres synthetized at CEA and dispersed in pure butanol with a concentration of $35 \mathrm{mg} / \mathrm{mL}$. Several suspensions were prepared with different particle sizes: $0.28,1,2.5$ and $10 \mu \mathrm{m}$.

\subsection{Characterization}

Fluidic behaviors were characterized by using two optical means: a fringe projection (moiré) technique [14] to determine surface topography and a contactless chromatic confocal sensor supplied by STIL (sensor serial number 1210-1042) to measure the liquid thickness on the ramp. The moiré pattern is composed of a straight-line grating with red and dark fringes, equi-spaced with a period of $7 \mathrm{~mm}$. 


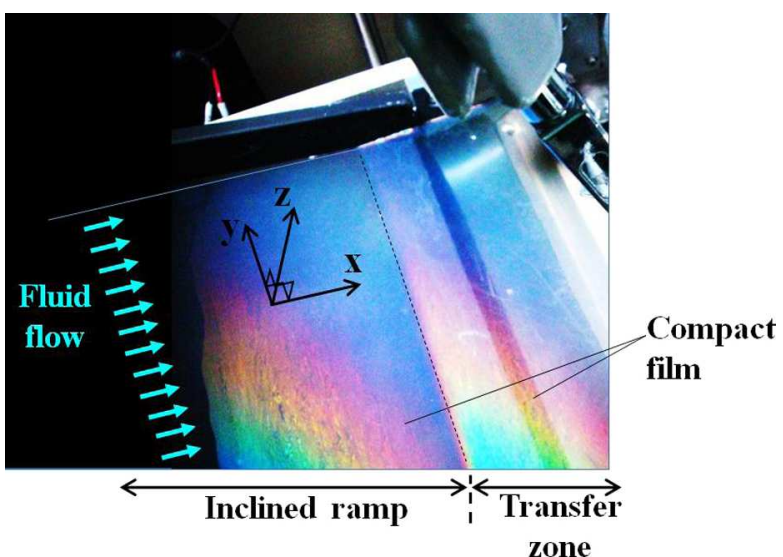

Fig. 4. Monolayer colloidal crystal of silica spheres $(\varnothing=1 \mu \mathrm{m})$ floating at the surface of the moving liquid (the colors arise from the diffraction of light).

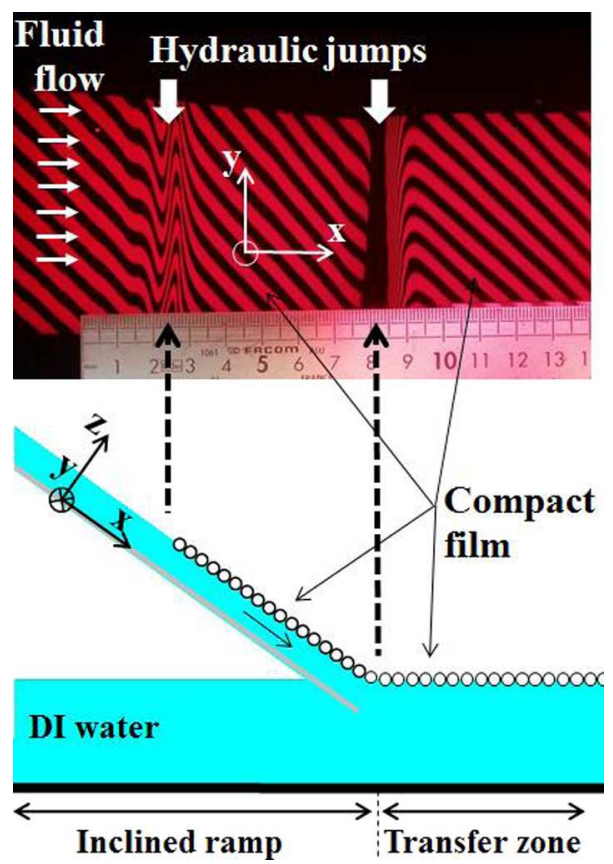

Fig. 5. Moiré pattern reflected by the moving liquid having a compact film of silica spheres floating at the surface $(\varnothing=1 \mu \mathrm{m})$.

The moiré pattern is projected normally to the transfer zone surface and covers the entire surface of the moving liquid. A camera aligned with the normal direction of the transfer zone captures the surface reflecting the projected fringe pattern. Figure 5 presents a view of the moiré pattern reflected by the liquid surface mirror showing specific deformations due to hydraulic jumps and slope of the liquid surface.

The fringe images are deformed when reflected by the curved liquid surface associated with the hydraulic jump, the fringe deformation being proportional to the local slope of the surface. This original experimental setup allowed us to get the surface profile in the jump region and to measure it along with the main process parameters (liquid flow rate, slope angle, temperature sensitive fluid properties such as dynamic viscosity or surface tension, particle sizes).

\section{Results and discussion}

\subsection{Simple models}

The liquid film is a low Reynolds number flow $(\operatorname{Re} \approx 100)$ but not a creeping flow. The velocity profile $V(z)$ in the thickness of the film is therefore a classical parabolic one that comes out integrating the 1D stationary simplification of the Navier-Stokes equation (a $x$-momentum balance equation):

$$
\mu \frac{\partial^{2} V(z)}{\partial z^{2}}=\frac{\partial p}{\partial x}=-\rho g \sin \alpha
$$

For the free surface liquid film, i.e., with no particle atop of it (Fig. 6), neglecting friction with air, integration is done with zero velocity at the contact of the $\operatorname{ramp}(z=0)$ and zero viscous stress at the top of the liquid $(z=h)$ :

$$
V(z)=\rho g \sin (\alpha) \mu^{-1}\left(h z-\frac{1}{2} z^{2}\right)
$$

The model is closed by integrating this velocity field in the thickness and equaling the volumetric liquid flow rate $Q$ (with a ramp width $L$ in the $y$-direction):

$$
Q=\frac{1}{3} \rho g \sin (\alpha) \mu^{-1} L h^{3}
$$

Therefore, the classical film thickness for a free surface is recovered:

$$
h=\left(\frac{3 \mu Q}{\rho g \sin (\alpha) L}\right)^{1 / 3}
$$

For the liquid film under the continuous particle monolayer, we assume that the upper boundary condition is: zero tangential velocity (i.e., in the $x$ and $y$ axis) and free normal velocity (i.e., in the $z$ axis).

This boundary condition is unusual, with no name associated to it. It is none of the classical Neumann, Dirichlet or Robin boundary condition. However, the experimental results thereafter will enforce this hypothesis. The tangential velocities are frozen whereas the normal velocity is free.

Under this hypothesis, the velocity profile goes to zero at $z=0$ and $z=h /$, the momentum balance equation (1) in the $x$-direction providing the rest of the factors:

$$
V(z)=\frac{1}{2} \rho g \sin (\alpha) \mu^{-1}\left(h_{z}^{\prime}-z^{2}\right)
$$

As previously, the imposed volumetric liquid flux $Q$ leads to:

$$
h^{\prime}=\left(\frac{12 Q}{\gamma L}\right)^{1 / 3}=\left(\frac{12 \mu Q}{\rho g \sin (\alpha) L}\right)^{1 / 3}
$$

The main consequence of this result is constant factor between $h$ and $h^{\prime}$

$$
\frac{h^{\prime}}{h}=2^{2 / 3} \approx 1.59
$$




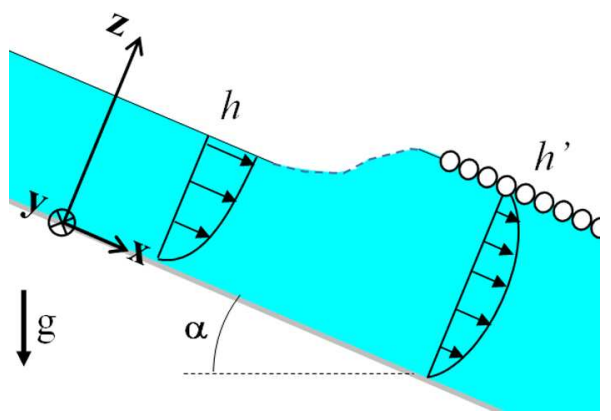

Fig. 6. Sketch of the first hydraulic jump connecting $h$ to $h$.

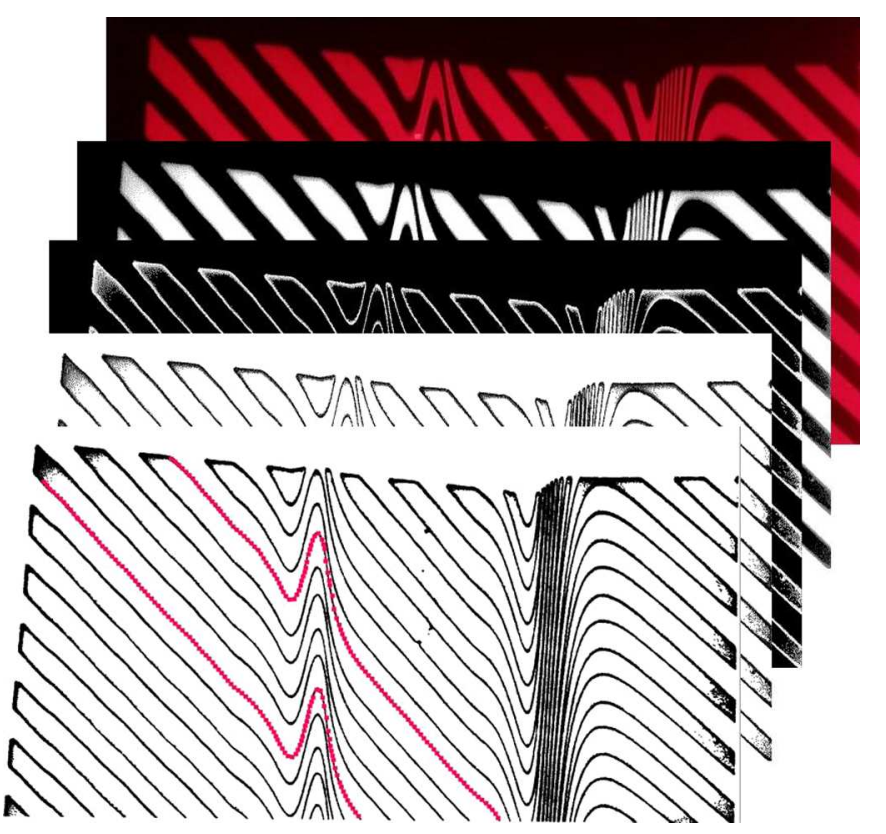

Fig. 7. Sketch of the contour extraction from the moiré projection images.

The viscous stress $\tau_{w}^{\prime}$ acting on the particle monolayer is deduced from the velocity profile:

$$
\begin{aligned}
\tau_{w}^{\prime} & =\left.\mu \frac{\partial V}{\partial z}\right|_{z=0 \& h^{\prime}}=\rho g \sin (\alpha) \frac{h^{\prime}}{2} \\
& =(\rho g \sin (\alpha))^{2 / 3}\left(\frac{3}{2} \frac{\mu Q}{L}\right)^{1 / 3}
\end{aligned}
$$

The different factors of this equation are potential lever to control and enhance the compaction of the monolayer. However, this viscous stress is several orders of magnitude lower than the capillary forces that should affect particles at the micrometer scale!

The length of the hydraulic jump is more difficult to establish properly. The height of this jump is $h /-h$, as calculated from equations (4) and (6). The length of the jump region involves the surface tension and will deserve a second paper to present the model. We give here only preliminary ideas of such a model (Figs. 6 and 7).

Using dimensional analysis of the jump region (somewhat analog to [15] or [16]), we can develop an approximate relation to predict the length $\lambda_{H J}$ of the hydraulic jump:

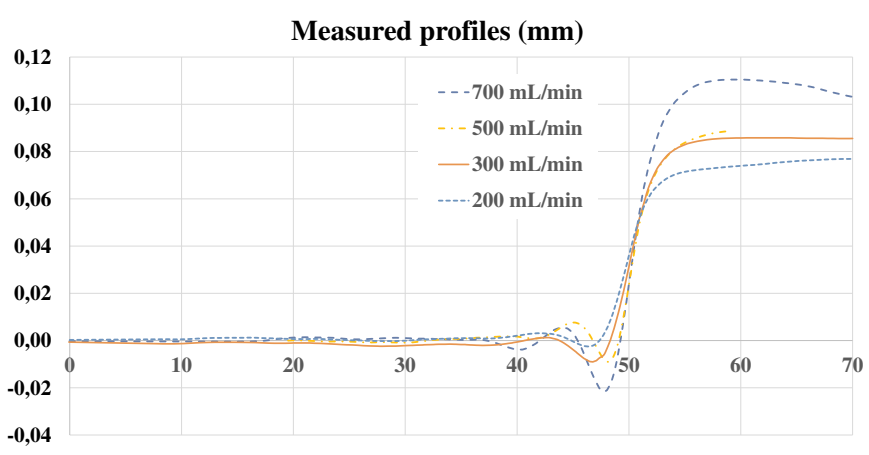

Fig. 8. $10 \mu \mathrm{m}$ particles measured profiles vs. flow rate $(z$ origin $=$ free surface).

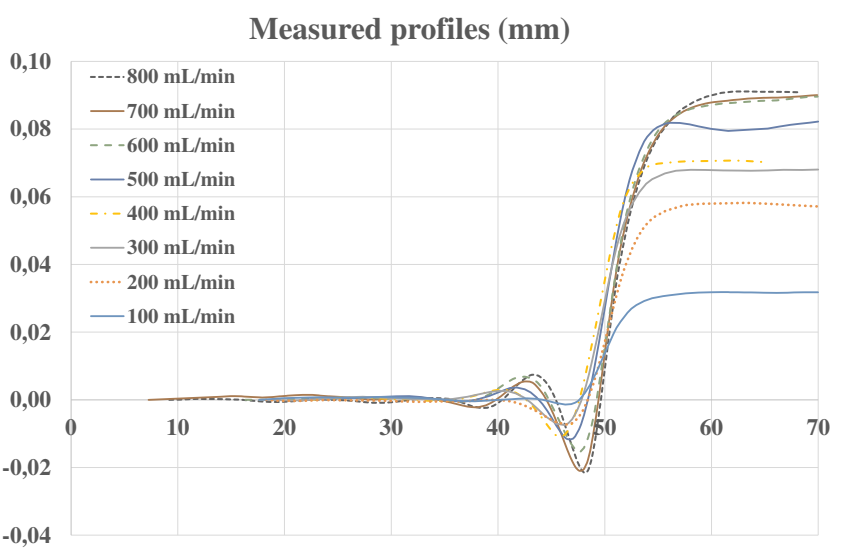

Fig. 9. $280 \mathrm{~nm}$ particles measured profiles vs. flow rate $(z$ origin $=$ free surface).

$$
\lambda_{H J}=\left(L \sigma\left(h^{\prime}-h\right)\right)^{1 / 3}(16 \mu Q)^{-1 / 3}\left(h+h^{\prime}\right)
$$

A rough estimate of this length for water, $\alpha=11^{\circ}$ and $500 \mathrm{~mL} \cdot \mathrm{min}^{-1}$ gives $3.0 \mathrm{~mm}$. However, the observed length (Figs. 7, 8 and 9) is longer than this value and is close to $20 \mathrm{~mm}$. This relation supposes that surface tension on the curvature of the profile has the same order of magnitude as the other terms. The detailed evaluation of the jump profile implies to revisit the local balance of the various forces (see, e.g., [17]). Along with $x$, the profile results from the equilibrium of three contributions at the interface:

- the viscous shear stress at the interface, $\tau_{\text {surf }}=\left.\mu \frac{\partial V}{\partial z}\right|_{z=\text { surf }}$,

- the hydreostatic contribution, $\rho g h \sin (\alpha)$,

- the surface tension contribution, $\sigma \kappa$.

\subsection{Measurements}

The moiré projection technique first uses a contour extraction on the moiré projection images. The rough images are first processed to sharpen the contrast between red and black. Then images are turned to pure black and white. Contours are extracted, filtered and then captured as $1 \mathrm{D}$ curves. The result is then interpreted into absolute 


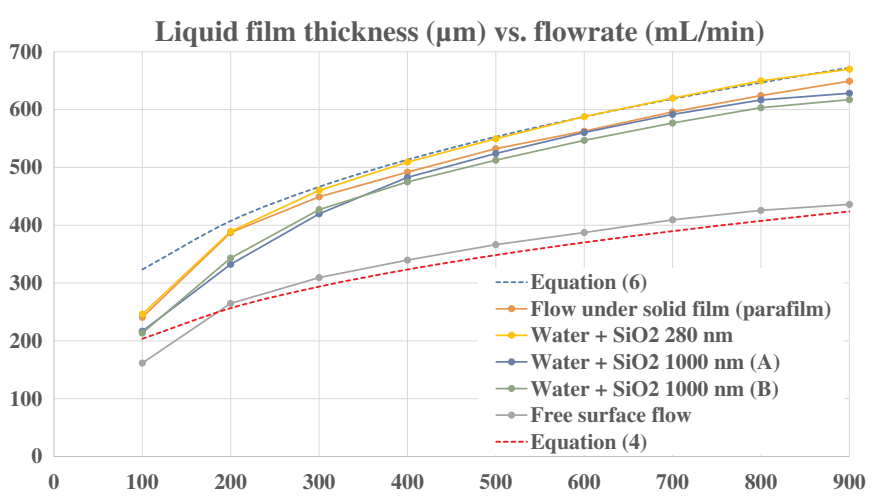

Fig. 10. Comparison between the measured film thicknesses and prediction of equations (4) and (6).
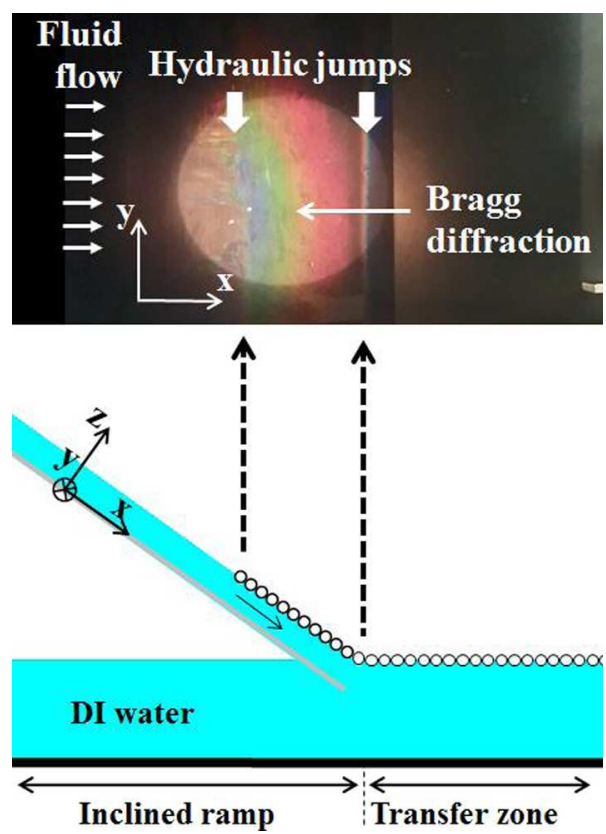

Fig. 11. View of the setup and a typical result of mode -1 of Bragg diffraction on the HCP lattice of particles. profiles by using simple optical reflection reconstruction. The large value of the distance from the fringes to the curved reflecting surface, and from this surface to the image acquisition, allows a magnification of the profile extension (100-200 $\mu \mathrm{m}$ are converted to several $\mathrm{cm})$.

The moiré technique allows more details than the sole height of the hydraulic jump. Figures 8 and 9 collect profile examples for different flow rates. As both $h$ and $h$ ' vary with flow rates, the curves use a common origin at the free surface profiles. It is noteworthy to look at the internal structure of the jump: one or two extrema are present in the profiles. It is possible to deduce from preliminary modeling of this zone (not fully reported here) that these extrema are linked to the surface tension acting on the interface.

The contactless chromatic confocal sensor provides absolute thickness measurement, contrary to the moiré technique. Measurements were performed on free surface film for various flow rates ranging from 100 to $900 \mathrm{~mL} \cdot \mathrm{min}^{-1}$.

The measured film thicknesses with a monolayer of silica particles (diameters of 280 and $1000 \mathrm{~nm}$ ), with a free surface flow and in the presence of a thin and continuous solid film of Parafilm ${ }^{\circledR}$ floating on the water, are reported in Figure 10. They clearly demonstrate that the hypothesis of frozen tangential velocities is valid. Model of equation (6) is well within the measurement for the flow rate range and the particle sizes we have explored.

Bragg diffraction of white light on the hexagonal closepacked (HCP) lattices was performed with collimated white light. Figure 11 shows a partial view of the setup and a typical result of mode -1 of Bragg diffraction on the HCP lattice of particles. Figure 12 gives the usual explanation of the location of modes in such diffraction. Figure 13 provides a typical result of mode -1 on $1 \mu \mathrm{m}$ particles.

By trying to measure the evolution of the isocontours on a series of images (from 100 to $900 \mathrm{~mL} \cdot \mathrm{min}^{-1}$ ), we finally succeed to show that these isocontours do not depend on the flow rate in the range of parameters we have investigated. The conclusion is simple: capillary forces that act on $1 \mu \mathrm{m}$ particles exceed by far the viscous shear stresses at the interface!

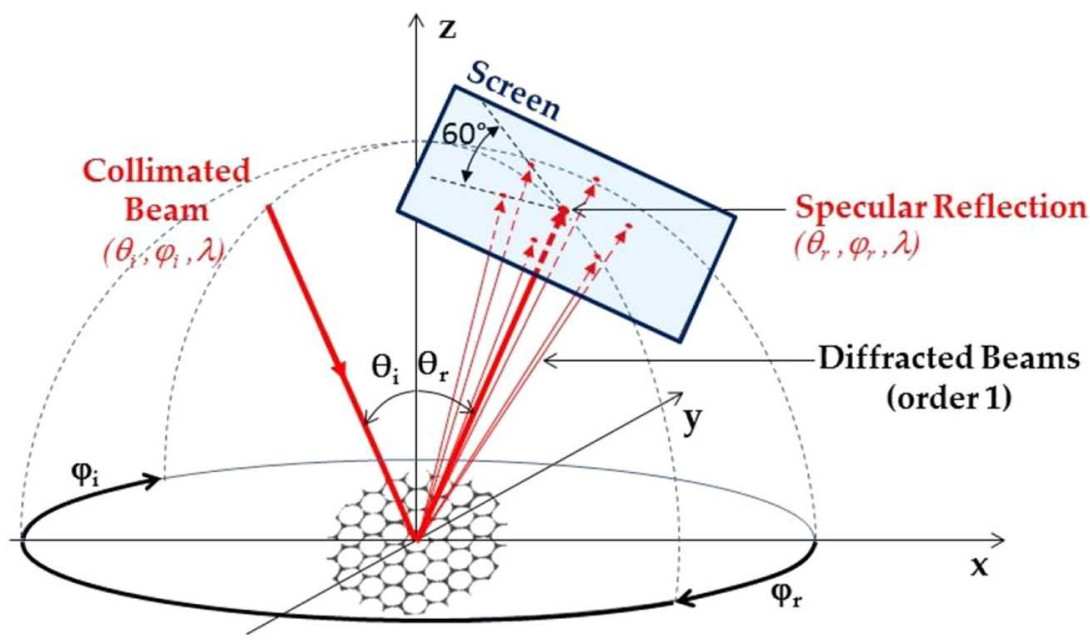

Fig. 12. Illustration of the diffraction by HCP structure at 0th and 1st order diffraction on a screen (from [12]). 


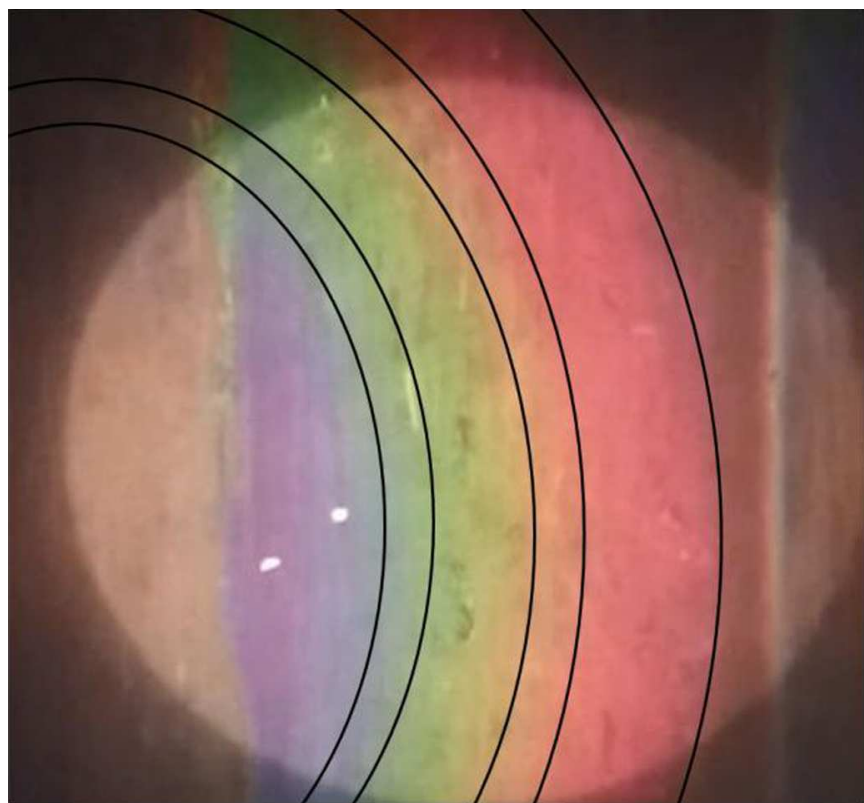

Fig. 13. Bragg diffraction on $1 \mu \mathrm{m}$ particle HCP: mode -1 and extraction of isocontours for various wave lengths $\left(500 \mathrm{~mL} \cdot \mathrm{min}^{-1}\right)$.

\section{Conclusion}

Colloidal self-assembly is a key bottom-up technique for surface structuring in a wide range of applications to control chemico-physical interactions at the micro/nanoscale. We presented an original process based on a liquid flowing down a ramp to assemble particles floating at the liquid interface. In presence of particles, the fluid flow accommodates and generates a hydraulic jump which can be characterized by optical means. We investigated several characteristics of these hydraulic jump using moiré technique and contactless sensor and compared the results with simple models. Concerning the profile, we observed specific curvatures depending on the liquid flow rate and particle diameters. However, rigorous modeling of these curvatures necessitates further theoretical and fine CFD investigations. Calculation of the height was in a good agreement with experimental results assuming that tangential velocities are frozen at the liquid under the continuous particle monolayer. Finally, Bragg diffraction analysis of floating HCP lattice constituted of $1 \mu \mathrm{m}$ particles has shown that capillary forces predominate over viscous shear stresses at the interface.

\section{Nomenclature}

$g \quad$ Gravity, $\mathrm{m} \cdot \mathrm{s}^{-2}$

$h \quad$ Film thickness in the free surface zone, $m$

h' Film thickness under the particle monolayer, $\mathrm{m}$

$L \quad$ Ramp width, i.e., in the $y$-direction (actual value: 29 $\mathrm{cm}), \mathrm{m}$

$p \quad$ Fluid pressure, $\mathrm{N} \cdot \mathrm{m}^{-2}$

$Q \quad$ Liquid film volume flow rate, $\mathrm{m}^{3} \cdot \mathrm{s}^{-1}$

$V \quad$ Liquid film velocity field, $\mathrm{m} \cdot \mathrm{s}^{-1}$ $x \quad$ Coordinate, parallel to the ramp surface, flow direction, $\mathrm{m}$

$y$ Coordinate, parallel to the ramp surface, width direction, $\mathrm{m}$

$z \quad$ Coordinate, perpendicular to the ramp surface, $\mathrm{m}$

$\alpha \quad$ Ramp angle ( $0^{\circ}$ is horizontal), -

$\kappa \quad$ Local curvature of the interface (along $x$-direction), $\mathrm{m}^{-1}$

$\lambda_{H J}$ Length of the hydraulic jump ( $x$-direction), m

$\mu \quad$ Dynamic viscosity, $\mathrm{Pa} \cdot \mathrm{s}$

$\rho \quad$ Liquid density, $\mathrm{kg} \cdot \mathrm{m}^{-3}$

$\sigma \quad$ Surface tension, $\mathrm{N} \cdot \mathrm{m}^{-1}$

$\tau_{w}^{\prime} \quad$ Shear stress, $\mathrm{Pa}$

\section{Acronyms}

CFD Computational fluid dynamics

DI Deionized

HCP Hexagonal close-packed lattice

MCCs Monolayer colloidal crystals

\section{References}

[1] Y. Li, W. Cai, G. Duan, Ordered micro/nanostructured arrays based on the monolayer colloidal crystals, Chem. Mater. 20 (2008) 615-624

[2] L. Grenet, F. Emieux, O. Delléa, A. Gerthoffer, G. Lorin, F. Roux, S. Perraud, Influence of coevaporation process on CIGS solar cells with reduced absorber thickness and current enhancement with periodically textured, glass substrates, Thin Solid Films 621 (2017) 188-194

[3] H. Wang, K.-Q. Zhang, Photonic crystal structures with tunable structure color as colorimetric sensors, Sensors 13 (2013) 4192-4213

[4] Z. Cai, N.L. Smith, J.T. Zhang, S.A. Asher, Twodimensional photonic crystal chemical and biomolecular sensors, Anal. Chem. 87 (2015) 5013-5025

[5] C. Park, K. Koh, U. Jeong, Structural color painting by rubbing particle powder, Scientific Reports 5, article number 8340, 2015

[6] C. Corbella, S. Portal, M. Rubio-Roy, M.A. Vallvé, J. IgnésMullol, E. Bertran, J.L. Andújar, Surface structuring of diamond-like carbon films by colloidal lithography with silica sub-micron particles, Diam. Relat. Mater. 19 (2010) 1124-1130

[7] M.C. Petty, Langmuir-Blodgett films: an introduction, Cambridge University Press, Cambridge 1996, ISBN: 0521413966

[8] A. Mihi, M. Ocaña, H. Miguez, Oriented colloidal-crystal thin films by spin-coating microspheres dispersed in volatile media, Adv. Mater. 18 (2006) 2244-2249

[9] B.G. Prevo, O.D. Velev, Controlled, rapid deposition of structured coatings from micro- and nanoparticle suspensions, Langmuir 20 (2004) 2099-2107

[10] T. Pinedo-Rivera, Assemblage convectif de colloïdes par forces de capillarité en milieu confiné: applications en plasmonique, Thèse, Université Grenoble I - Joseph Fourier, 2009 
[11] K. Askar, S.-Y. Leo, C. Xu, D. Liu, P. Jiang, Rapid electrostatics-assisted layer-by-layer assembly of near-infrared active colloidal photonic crystals, J. Colloid Interface Sci. 482 (2016) 89-94

[12] O. Delléa, O. Shavdina, P. Fugier, P. Coronel, E. Ollier, S.-F. Désage, Control methods in microspheres precision assembly for colloidal lithography, IFIP Adv. Inform. Commun Technol. 435 (2014) 107-117

[13] http://wenku.baidu.com/view/9e4c5e8dcc22bcd126ff0cad. html?from $=$ search
[14] K. Creath, J.C. Wyant, Moiré and fringe projection techniques, in: Optical shop testing, Daniel Malacara, 2nd edn., John Wiley \& Sons, New York, 1992, ISBN: 0471522325

[15] J.H. Snoeijer, G. Delon, M. Fermigier, B. Andreotti, Avoided critical behavior in dynamically forced wetting, Phys. Rev. Lett. 96 (2006) 174504-1-174504-4

[16] P. Aussillous, D. Quéré, Quick deposition of a fluid on the wall of a tube, Phys. Fluids 12 (2000) 2367-2690

[17] A. Oron, S.H. Davis, S.G. Bankoff, Long-scale evolution of thin liquid films, Rev. Mod. Phys. 69 (1997) 931-980

Cite this article as: O. Delléa, O. Lebaigue, Boostream: a dynamic fluid flow process to assemble nanoparticles at liquid interface, Mechanics \& Industry 18, 602 (2017) 